\title{
On the response of quasi-adiabatic particles to magnetotail reconfigurations
}

\author{
Dominique C. Delcourt ${ }^{1}$, Helmi V. Malova ${ }^{2}$, and Lev M. Zelenyi ${ }^{2}$ \\ ${ }^{1}$ LPP, Ecole Polytechnique-CNRS-UPMC, Paris, France \\ ${ }^{2}$ Space Research Institute, Russian Academy of Sciences, Moscow, Russia \\ Correspondence to: Dominique C. Delcourt (dominique.delcourt@ 1pp.polytechnique.fr)
}

Received: 9 September 2016 - Revised: 7 December 2016 - Accepted: 8 December 2016 - Published: 3 January 2017

\begin{abstract}
We investigate the response of quasi-adiabatic particles to dynamical reconfigurations of the magnetotail field lines. Although they travel through a sharp field reversal with a characteristic length scale smaller than their Larmor radii, these quasi-adiabatic particles experience a negligible net change in magnetic moment. We examine the robustness of such a quasi-adiabatic behavior in the presence of a large surging electric field induced by magnetic field line reconfiguration as observed during the expansion phase of substorms. We demonstrate that, although such a short-lived electric field can lead to substantial nonadiabatic heating, quasi-adiabaticity is conserved for particles with velocities larger than the peak $\boldsymbol{E} x \boldsymbol{B}$ drift speed. Because of the timevarying character of the magnetic field, it is not possible to use the adiabaticity parameter $\kappa$ in a straightforward manner to characterize the particle behavior. We rather consider a $\kappa$ parameter that is averaged over equatorial crossings. We demonstrate that particles intercepting the field reversal in the early stage of the magnetic transition may experience significant energization and enhanced oscillating motion in the direction normal to the midplane. In contrast, particles interacting with the field reversal in the late stage of the magnetic transition experience weaker energization and slower oscillations about the midplane. We show that quasi-adiabatic particles accelerated during such events can lead to energy-time dispersion signatures at low altitudes as is observed in the plasma sheet boundary layer.
\end{abstract}

Keywords. Space plasma physics (charged particle motion and acceleration)

\section{Introduction}

Charged particles traveling in a sharp field reversal may not conserve their magnetic moment (first adiabatic invariant) because of significant variations of the magnetic field within a cyclotron turn. A parameter that is widely used to characterize this nonadiabatic behavior is the parameter $\kappa$, introduced by Büchner and Zelenyi (1989) and defined as $\kappa=\left(R_{\mathrm{C}} / \rho_{\mathrm{L}}\right)^{1 / 2}$, where $R_{\mathrm{C}}$ is the minimum field line curvature radius and $\rho_{\mathrm{L}}$ is the maximum Larmor radius of the particle. For $\kappa$ greater than 3, the particle motion is adiabatic and the guiding center approximation (e.g., Northrop, 1963) is valid. For $\kappa$ between 3 and 1, the motion may become chaotic (Büchner and Zelenyi, 1989) and pitch angle scattering can lead to prominent filling of the loss cone (Sergeev et al., 1983). In this $1<\kappa<3$ range, deviation from adiabaticity may be described by considering a perturbation of the particle gyro-motion with a centrifugal impulse (Delcourt et al., 1996). As a result of this centrifugal perturbation, damping or enhancement of the particle magnetic moment (denoted by $\mu$ hereinafter) may be obtained depending upon pitch angle and gyration phase; hence, in the long term, this may result in a possibly chaotic behavior with prominent dependence upon initial conditions (see also Anderson et al., 1997). For $\kappa$ smaller than 1, a distinct dynamical regime is obtained since the particle Larmor radius becomes larger than the field line curvature radius and the particle may oscillate on either side of the field reversal midplane.

In this latter $\kappa<1$ dynamical regime, distinct classes of particle orbits have been uncovered (e.g., Chen and Palmadesso, 1986; Büchner and Zelenyi, 1989). The Speiser (equivalently, "transient") orbit that was put forward in the pioneering work of Speiser (1965) forms one of these classes 

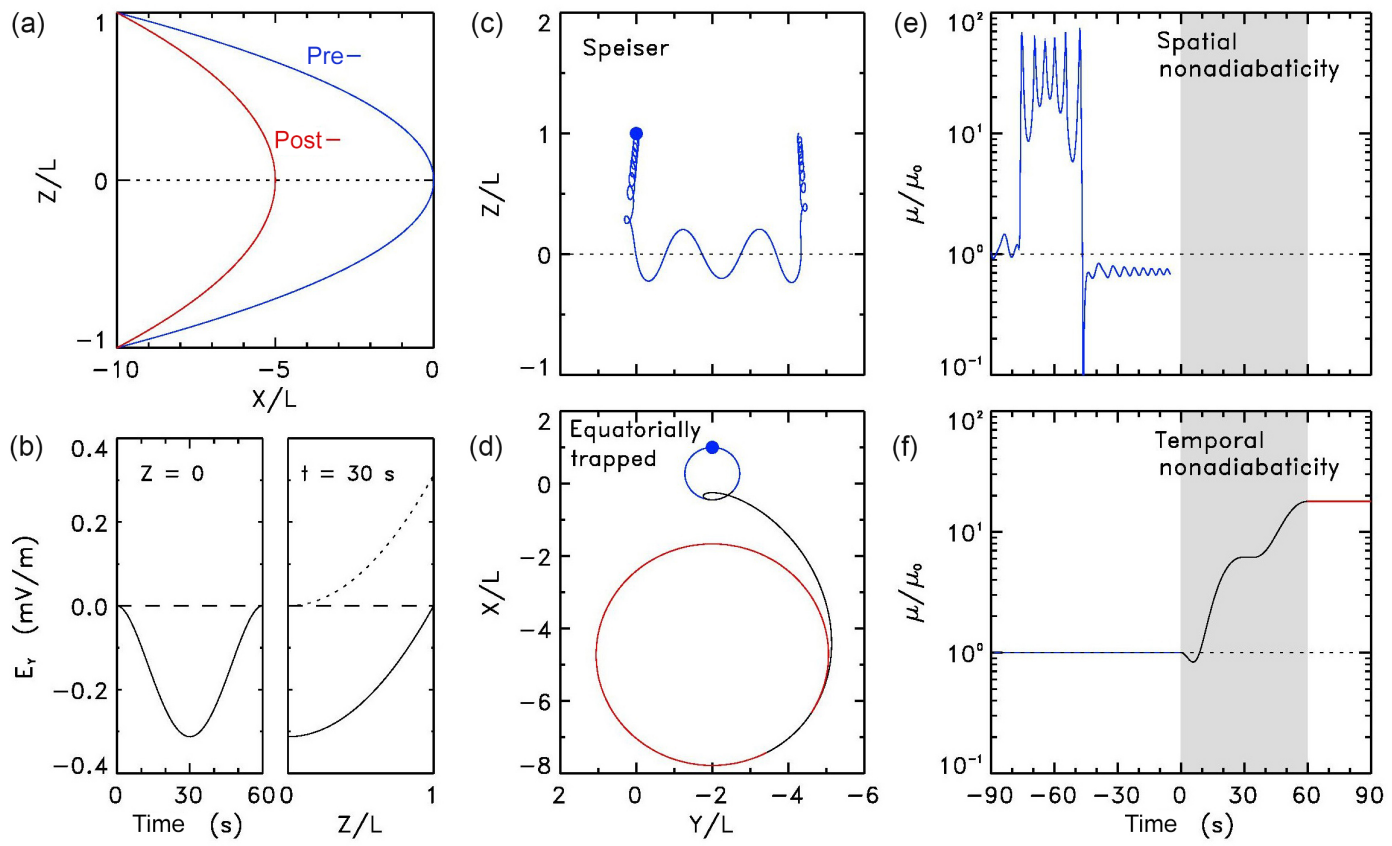

Figure 1. (a) Model magnetic field lines (Eq. 3) in the pre- and post-dipolarization configurations (coded in blue and red, respectively). (b) Total electric field $(\boldsymbol{E}=-\partial \boldsymbol{A} / \partial t+\nabla \Phi$; solid line) at $Z=0$ as a function of time and at $t=30 \mathrm{~s}$ as a function of $Z$ height. The dotted line shows the purely induced electric field $\boldsymbol{E}=-\partial \boldsymbol{A} / \partial t$. Model trajectory and magnetic moment versus time of (c and $\mathbf{e})$ quasi-adiabatic $\mathrm{H}^{+}$and $\left(\mathbf{d}\right.$ and $\mathbf{f}$ ) equatorially trapped $\mathrm{H}^{+}$(blue and red relate to pre- and post-dipolarization configurations, respectively). A $60 \mathrm{~s}$ magnetic transition (shaded area) is considered. Full circles show the initial $\mathrm{H}^{+}$positions.

whereby the net $\mu$ change experienced by the particles after crossing of the field reversal is negligible. Accordingly, Speiser particles that originate from regions of strong magnetic field may return to such regions after transport into the magnetotail. Although the motion of these Speiser particles differs from a regular helical motion during oscillation about the midplane, it was shown by Büchner and Zelenyi (1989) that their meandering motion does have some regularity with the action integral $I_{Z}=\int P_{Z} \mathrm{~d} Z$ (where $Z$ is the direction normal to the midplane and $P_{Z}$ is the generalized momentum) as an approximate invariant, hence the denomination of these Speiser particles as "quasi-adiabatic". This meandering motion is due to the rapid change in the $B_{X}$ component (where $X$ points in the Sun-Earth direction) above and below the midplane. Also, because of the small $B_{Z}$ component of the magnetic field, these meandering particles are gradually turned back from tailward to earthward directions of propagation and the current that is carried across the magnetotail during this motion critically depends upon particle species (e.g., Malova et al., 2013). Other classes of orbits in the $\kappa<1$ regime are quasi-trapped particles that experience repeated crossings of the midplane with significant $\mu$ changes, and trapped particles such as those that mirror in the equatorial plane.

As for Speiser particles, it was shown by Chen and Palmadesso (1986) that this behavior is more pronounced for specific values of the $\kappa$ parameter (or, equivalently, of the dimensionless Hamiltonian), a feature that was interpreted as the result of resonance between the fast oscillations in the $Z$ direction and the slow gyro-motion in the $X-Y$ plane ( $Y$ pointing in the dusk-dawn direction). Burkhart and Chen (1991) examined further this resonance effect and obtained the following empirical relationship to characterize the $N$ th resonance $(N$ being an integer $\geq 1$ ):

$\kappa_{N} \approx \frac{0.8}{N+0.6}$.

A better fit to numerical simulations of resonances was obtained by Delcourt and Martin (1999; see their Eq. 16), viz.,

$\kappa_{N} \approx \frac{0.775}{N+0.456}$.

According to Eq. (2), the first resonance $(N=1)$ is obtained for $\kappa_{1} \approx 0.53$ with orbits that feature two crossings of the midplane. The second resonance $(N=2)$ is obtained for $\kappa_{2} \approx 0.316$ with orbits that feature three crossings of the midplane, and so on. This resonance effect can lead to the formation of "beamlets" that propagate along the magnetic field lines and lead to localized velocity-dispersed structures at low altitudes (e.g., Ashour-Aballa et al., 1995).

The dynamics of Speiser (quasi-adiabatic) particles has been examined in a variety of studies that generally considered one-dimensional (in the $Z$ direction) steady-state situations. In such situations, the dawn-to-dusk convection 
electric field can be removed via transformation to the de Hoffman-Teller frame. In this reference frame that moves at the equatorial $\boldsymbol{E} x \boldsymbol{B}$ drift speed, the convection electric field vanishes so that equations of motion can be written in a simple manner. In the present study, we investigate the response of quasi-adiabatic particles in the presence of a timevarying magnetic field. A characteristic feature of the expansion phase of substorms at Earth is the dipolarization of magnetic field lines that takes place within a few minutes and propagates both radially and azimuthally within tens of minutes (see, e.g., Miyashita et al., 2009, and references therein). Such rapidly propagating dipolarization events (e.g., Reeves et al., 1996; Sarris et al., 2002; Runov et al., 2009) occur in other magnetospheric environments as well but with different characteristic timescales, for instance of the order of seconds at Mercury (e.g., Sundberg et al., 2012). The rapidly changing magnetic field during such events gives rise to an induced electric field that can reach a magnitude of several millivolt per meter (e.g., Artemyev et al., 2015). The early observations of Aggson et al. (1983) provide a prototypical example of such large electric fields induced by magnetic field line dipolarization. On top of the mean electric field variation, fluctuations on short timescales also are observed, as examined for instance by Nosé et al. (2014). Under the effect of these large induced electric fields, ions may be subjected to prominent acceleration as examined in a variety of studies (e.g., Delcourt et al., 1990; Zhou et al., 2010; Ukhorskyi et al., 2013; Artemyev and Vasiliev, 2015; see review by Birn et al., 2012).

In the present study, we are interested in the response of quasi-adiabatic particles to such dipolarization events, focusing on the mean field variation. Indeed, it cannot be taken for granted that Speiser particles persist during short-lived dipolarization since the $B_{X}$ component of the magnetic field that is responsible for the fast meandering motion varies on a timescale comparable to the slow gyro-motion (of the order of a few minutes). Also, because the electric field induced by the magnetic transition rapidly varies with time, it is not possible to use transformation to the de Hoffman-Teller frame. The use of the adiabaticity parameter $\kappa$ proves to be problematic as well since magnetic field lines are turning more dipolar and their curvature rapidly decreases with time. In the following, we first present the simple dipolarization model that was used as well as some typical particle trajectories. We then examine the response of quasi-adiabatic particles to field line dipolarization in a more systematic manner.

\section{Modeling of magnetotail reconfiguration}

To examine the dynamics of quasi-adiabatic particles during short-lived reconfigurations of the distant magnetotail, we consider the simple parabolic field defined as

$\boldsymbol{B}(X, Z)=B_{\mathrm{t}} \frac{Z}{L} \boldsymbol{x}+B_{\mathrm{n}} z$. here, $L$ is a reference scale length representing the half thickness of the current sheet and $\boldsymbol{x}$ and $\boldsymbol{z}$ are unit vectors in the $X$ and $Z$ directions, respectively (with $X$ axis pointing tailward and $Z$ axis pointing from south to north). Also, $B_{\mathrm{n}}$ is the small magnetic field component normal to the midplane. In the present study, the rapid magnetic transition is modeled via the tail lobe field $B_{\mathrm{t}}$ in Eq. (3) that we define as

$B_{\mathrm{t}}(t)=B_{\text {pre- }}+f(t)\left(B_{\text {post- }}-B_{\text {pre- }}\right)$.

In Eq. (4), $B_{\text {pre- }}$ and $B_{\text {post- }}$ are the tail lobe field magnitudes before and after the reconfiguration, respectively. Also, $f(t)$ is a polynomial that describes the rate of change in the magnetic field. More specifically, imposing a zero induced electric field as well as its zero slope at the onset and at the end of the magnetic transition leads to a polynomial of degree 5 for $f(t)$, as described in Appendix A of Delcourt et al. (1990) (see Eq. A8 of that paper). Denoting by $\tau_{B}$ the timescale of the reconfiguration, this polynomial smoothly varies between 0 at $t=0$ and 1 at $t=\tau_{B}$.

Note that, at low latitudes, field line dipolarization may be characterized by either an increase in $B_{Z}$, equivalently $B_{\mathrm{n}}$ in Eq. (3) (e.g., Runov et al., 2009) or a decrease in $B_{X}$ as displayed in Aggson et al. (1983) (see Fig. 4 of that paper). In the present study, we model the field line dipolarization via a decrease in $B_{\mathrm{t}}$ in Eq. (4), from $B_{\text {pre- }}=20$ to $B_{\text {post- }}=10 \mathrm{nT}$, the other parameters $B_{\mathrm{n}}$ and $L$ in Eq. (3) being kept constant (set to $1 \mathrm{nT}$ and $0.3 R_{\mathrm{E}}$, respectively). For the field line curvature radius that is written as $R_{\mathrm{C}}=B_{\mathrm{n}} L / B_{\mathrm{t}}$ at $Z=0$, one then obtains an increase by a factor of 2 , qualitatively consistent with the curvature radius evolution expected during dipolarization.

As for the electric field, it was considered to be the sum of two contributions. The first contribution is that induced by the time-varying magnetic field, viz., $\boldsymbol{E}=-\partial \boldsymbol{A} / \partial t$ where the vector potential $\boldsymbol{A}$ is such that $\operatorname{curl}(\boldsymbol{A})=\boldsymbol{B}$ as given by Eq. (3). Here, we choose for this vector potential $A_{Y} \propto Z^{2}$. The induced electric field obtained (in the $Y$ direction) depends upon the mathematical form arbitrarily adopted for A, and this electric field alone is thus not sufficient to fully characterize the motion of the magnetic field lines, as described in Appendix B of Delcourt et al. (1990). For this purpose, we also impose that the $\boldsymbol{E} x \boldsymbol{B}$ drift speed vanishes at $Z=L$, and to do so, a second contribution, $\nabla \Phi$, is taken into account as well. As will be made more apparent in Fig. 1, the gauge $\Phi$ that we introduce here only has a component in the $Y$ direction and a constant value at given time $t$, hence a total electric field $\boldsymbol{E}=-\partial \boldsymbol{A} / \partial t+\nabla \Phi$ that maximizes at $Z=0$ and vanishes at $Z=L$. For simplicity, the steady-state dawn-to-dusk (in the $-Y$ direction) convection electric field was not considered in the following computations. The time evolution of the magnetic field can be seen in the upper leftmost panel of Fig. 1, which shows field lines prior to (coded in blue) and after (coded in red) the magnetic transition. It can clearly be seen here that the magnetic field line evolves from a stretched 
Z

(a)

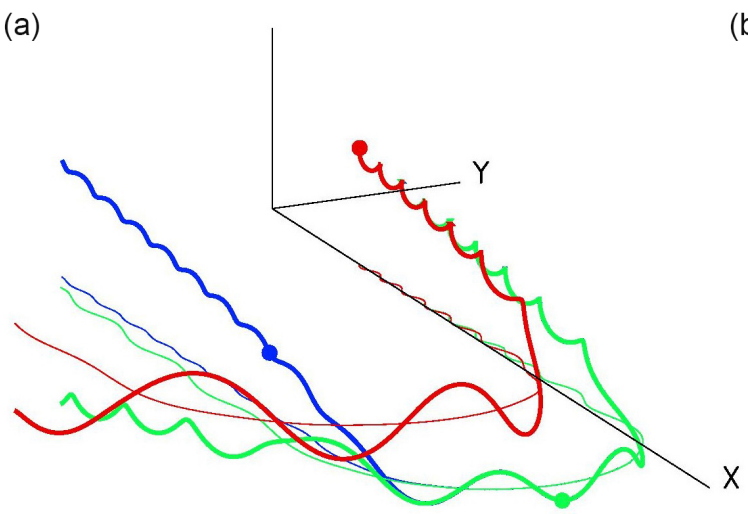

(b)
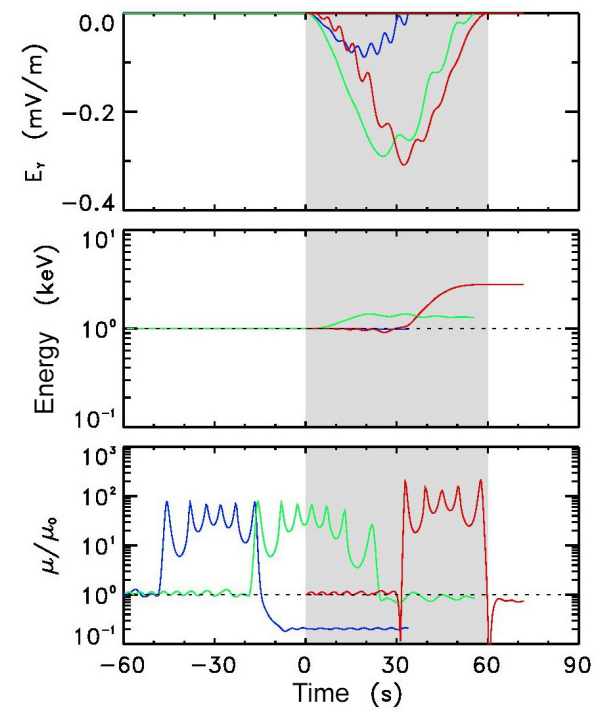

Figure 2. Left: three-dimensional trajectories of test $\mathrm{H}^{+}$launched at different (color-coded) times from $Z=L$. Thin lines show the $\mathrm{H}^{+}$ trajectory projection in the equatorial plane. Right: color-coded electric field, energy, and magnetic moment versus time.

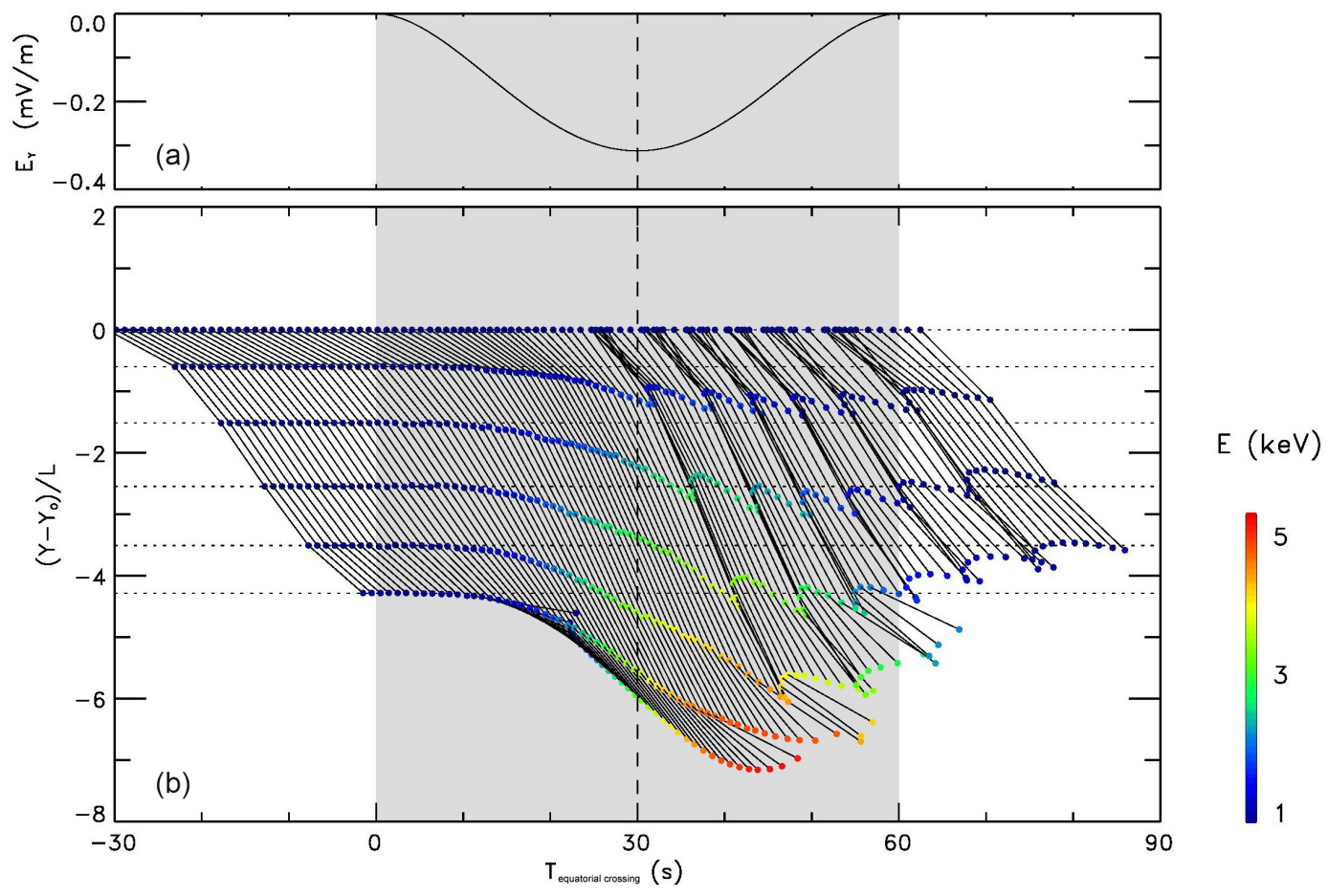

Figure 3. Bottom: $Y$ locations of $\mathrm{H}^{+}$equatorial crossings as a function of equatorial crossing time. The $Y$ coordinate is evaluated with respect to the first midplane crossing and normalized to the half thickness of the current sheet, $L$. The instantaneous $\mathrm{H}^{+}$energy is coded according to the color scale on the right. Top: electric field as a function of time. A $60 \mathrm{~s}$ (shaded area) magnetic transition is considered.

configuration to a more dipolar one. The electric field variations during this magnetic transition can be seen in the lower leftmost panels of Fig. 1. On the left, it can be seen that the total electric field at $Z=0$ points in the $-Y$ direction and reaches a peak magnitude of $\sim 0.3 \mathrm{mV} \mathrm{m}^{-1}$ at half collapse. On the right, it is apparent from the $Z$ profile obtained at half collapse that the total electric field (solid line) maximizes at $Z=0$ and vanishes at $Z=L$, due to the combined effect of 


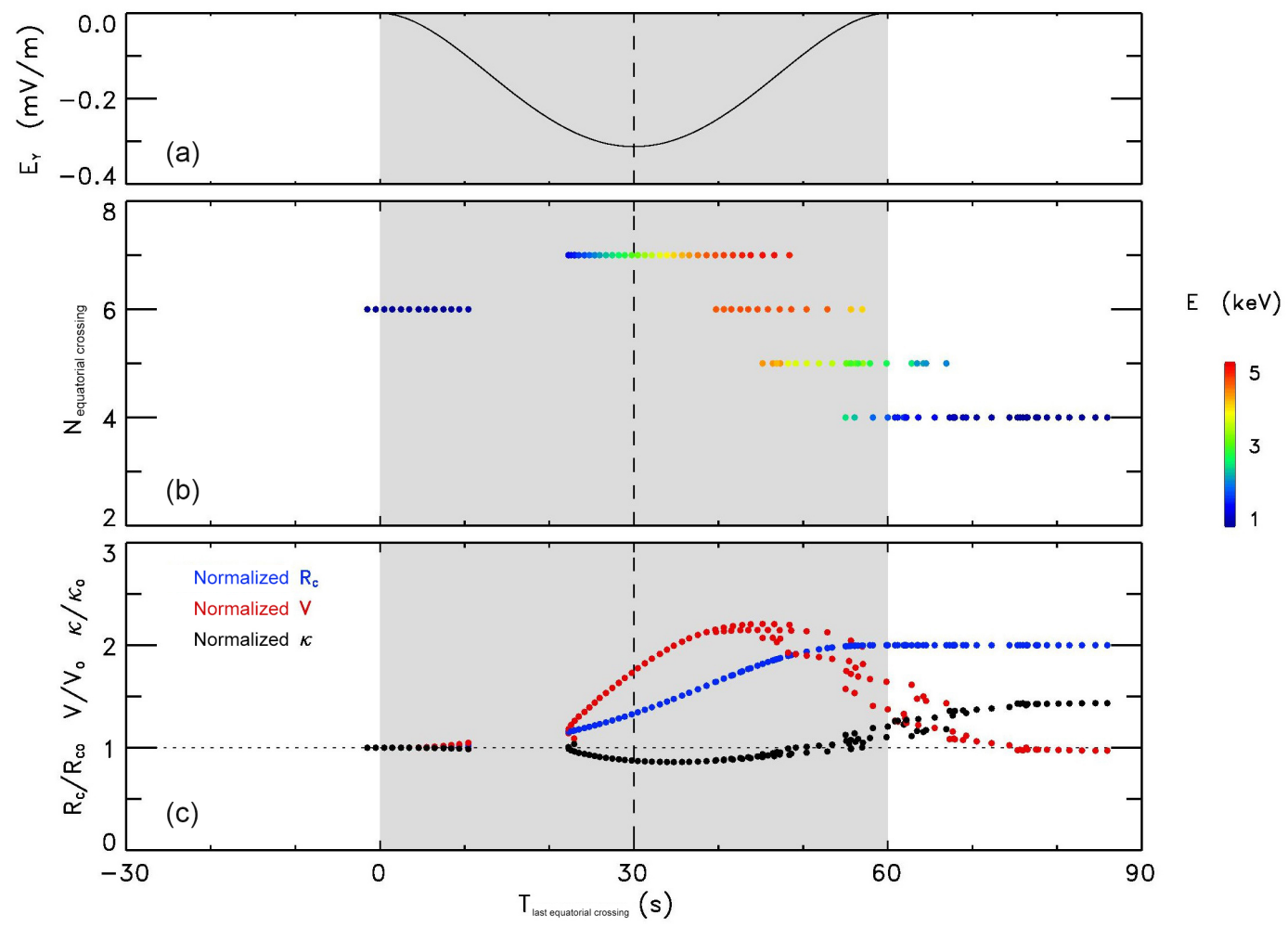

Figure 4. From top to bottom: electric field magnitude, number of equatorial crossings, and final value of $\kappa$ (normalized to the initial one; coded in black) as a function of last equatorial crossing time. The bottom panel also shows the final values of field line curvature radius and particle speed (coded in blue and red, respectively), normalized to their initial values. In the middle panel, the final $\mathrm{H}^{+}$energy is coded according to the color scale on the right.

the purely induced electric field (dotted line) and of the gauge transformation.

The upper right panels of Fig. 1 show an example of particle orbits obtained in the initial stretched configuration. As mentioned above, because of the sharp field reversal, the particle motion near the midplane differs from a regular helical motion and the trajectory shown here actually provides an example of Speiser orbit obtained at $N=5$ resonance $(\kappa \approx 0.142$ in Eq. 2), as portrayed in Fig. 3 of Speiser (1965). Although nonadiatic per se, the particle motion about the midplane does have some regularity, and it can be seen in the upper rightmost panel that the net $\mu$ change at the exit of the neutral sheet is negligible, hence the denomination of such particles as quasi-adiabatic. While the upper panels show an example of spatial nonadiabaticity (i.e., due to spatial variation of the magnetic field on the length scale of the particle Larmor radius), the bottom panels of Fig. 1 show an example of temporal nonadiabaticity (i.e., due to temporal variation of the magnetic field on the timescale of the particle cyclotron period). More specifically, the test $\mathrm{H}^{+}$considered in these bottom panels is trapped in the equatorial plane so that there is no parallel motion and associated violation of the first adiabatic invariant upon crossing of the sharp field reversal. In these lower panels, we assume that the magnetic field evolves from a stretched configuration to a more dipolar one on a timescale of $1 \mathrm{~min}$ (e.g., Aggson et al., 1983). It is clearly apparent from these lower panels that the electric field induced by the magnetic transition leads to a rapid enhancement of the particle magnetic moment by more than 1 order of magnitude; hence, there is a significantly larger Larmor radius after dipolarization.

Figure 1 demonstrates that particles may behave nonadiabatically either because of spatial variations of the magnetic field or because of temporal ones. The question that we wish to address in the present study has to do with a combination of these two types of nonadiabaticity. That is, how do quasiadiabatic (Speiser) particles respond to a short-lived reconfiguration of the magnetic field lines and to the associated impulsive electric field?

\section{Model results}

The above time-varying parabolic field model (Eqs. 3-4) was used to perform some parametric exploration of particle dynamics during reconfiguration of the magnetotail. As in Delcourt et al. (1990), the instantaneous magnetic moment of the particles in this parametric exploration was calculated in the reference frame moving at the $\boldsymbol{E} \times B$ drift speed, i.e., 

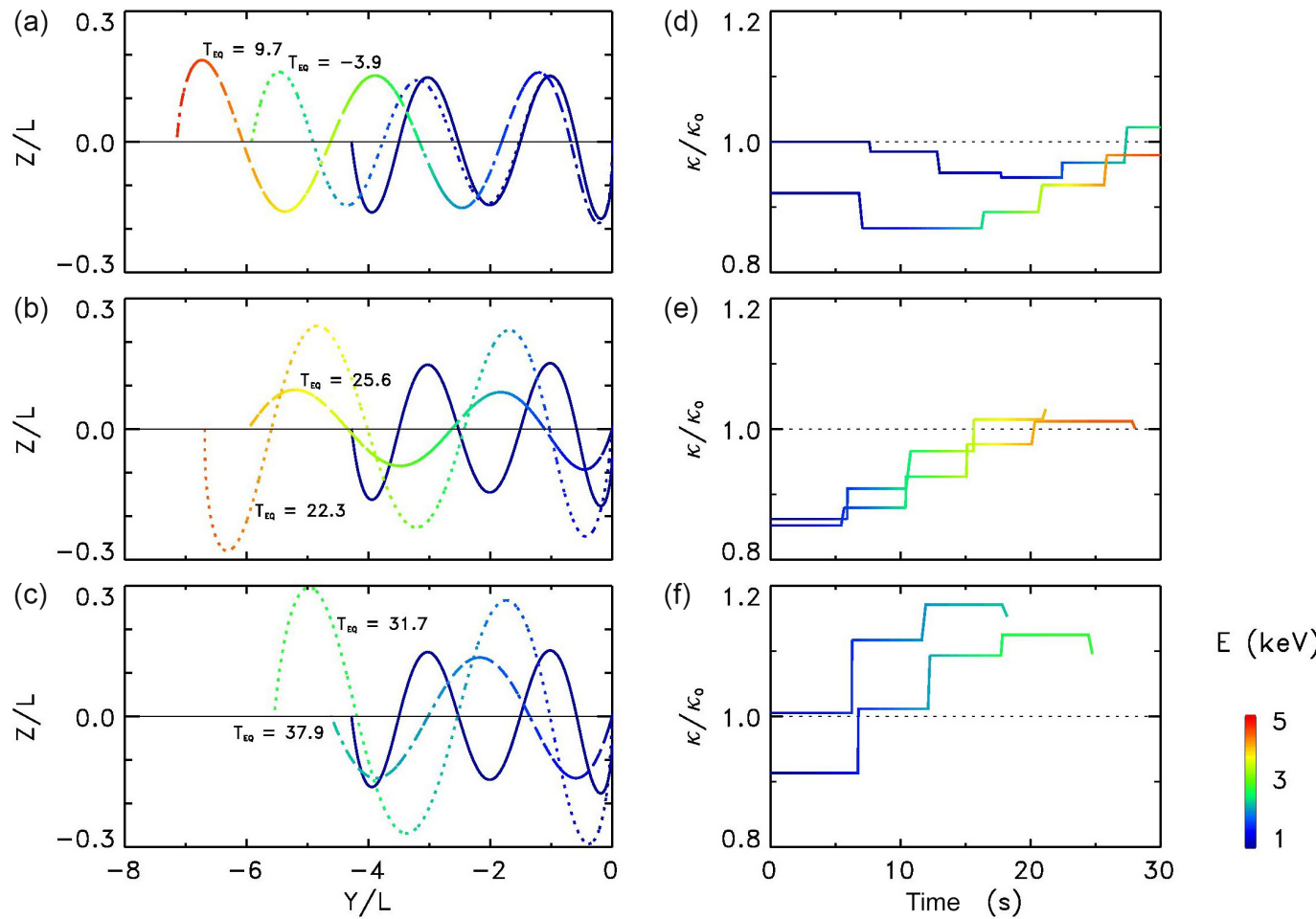

Figure 5. From top to bottom in left panels: meandering trajectory sequences during field line dipolarization for different equatorial crossing times (the time of the first equatorial crossing is indicated for each trajectory). The $\mathrm{H}^{+}$trajectory shown in blue is the reference trajectory in the pre-dipolarization configuration. From top to bottom in right panels: corresponding $\kappa$ parameters (normalized to the value in the pre-dipolarization configuration) as a function of time. The instantaneous $\mathrm{H}^{+}$energy is coded according to the color scale on the right.

$\mu=m\left(\boldsymbol{V}_{\perp}-\boldsymbol{V}_{\boldsymbol{E} \times \boldsymbol{B}}\right)^{2} / 2 B$, where $m$ is the particle mass and $B$ the magnetic field magnitude (e.g., Northrop, 1963; Cary and Brizard, 2009). As will be discussed in detail hereinafter, this magnetic moment is not conserved during the particle meandering sequence near $Z=0$, but the issue of interest here is to examine the net change in $\mu$, viz., to compare $\mu$ values at entry and at the exit of the field reversal (equivalently, before and after the meandering sequence). Figure 2 shows examples of trajectories obtained for different initial conditions considering a $60 \mathrm{~s}$ relaxation from tail-like to dipolelike configuration. Here, test $\mathrm{H}^{+}$at $N=5$ resonance (i.e., an initial energy of $\sim 1.1 \mathrm{keV}$ with the present model parameters; see Eq. 2) was launched at different times from $Z=L$. Because of these different injection times, the particles are struck by dipolarization at distinct points of their motion. It can be seen in the leftmost panel of Fig. 2 that the test $\mathrm{H}^{+}$ coded in blue travels in the pre-dipolarization configuration and executes six crossings of the midplane. Since for simplicity there is no electric field other than that induced by the time-varying magnetic field, no energy gain is obtained for this particle upon interaction with the field reversal. By comparison, the test $\mathrm{H}^{+}$coded in green in Fig. 2 that is struck by dipolarization during the meandering sequence near $Z=0$ experiences an additional crossing of the midplane. This particle exits the field reversal with a net energy gain of a few hundred electronvolt and with a magnetic moment nearly identical to the initial one.

It is important here to keep in mind the definition of quasi-adiabaticity, as described in the introduction. That is, this quasi-adiabatic behavior concerns particles that meander above and below the midplane and for which one has $\kappa<1$. For these particles, the magnetic moment is not conserved during the meandering sequence, but a new invariant (the action integral $I_{Z}$; see above) can be defined throughout the motion. The term "quasi-adiabatic" is a steady-state concept that was introduced by Büchner and Zelenyi (1989) to emphasize the fact that, at the exit of the field reversal, these particles have a magnetic moment nearly identical to that at entry. In other words, the net change in magnetic moment after interaction with the field reversal is negligible, despite the nonadiabatic meandering sequence. The trajectory coded in green in Fig. 2 provides an example of such behaviors and demonstrates that quasi-adiabaticity may persist during dipolarization although the particle is struck by a rapidly changing electric field.

As for the test $\mathrm{H}^{+}$coded in red in Fig. 2, it is launched somewhat later than the other $\mathrm{H}^{+}$and it intercepts the midplane during the late phase of the magnetic transition. At this time, the field line curvature radius has significantly increased so that the timescale of the $Z$ oscillation about the 

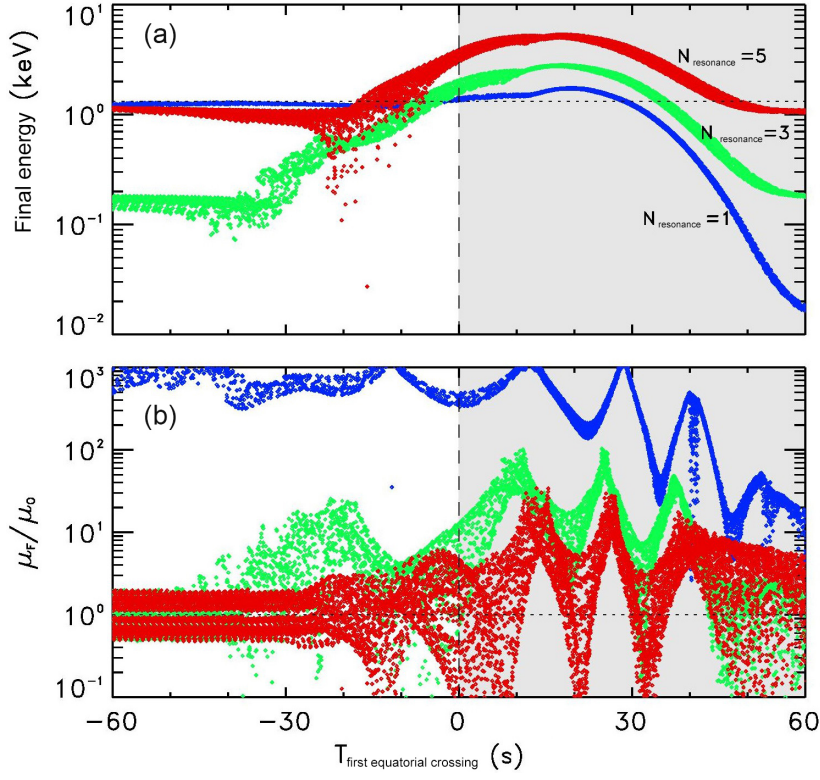

Figure 6. Final $\mathrm{H}^{+}$energy (top) and magnetic moment (bottom) as a function of the first equatorial crossing time. Different initial energies are considered ( $N=1, N=3$, and $N=5$ in Eq. (2), coded in blue, green, and red, respectively). A $60 \mathrm{~s}$ (shaded area) magnetic transition is considered.

midplane (which varies as $R_{\mathrm{C}} / V$, where $R_{\mathrm{C}}$ is the field line curvature radius and $V$ is the particle speed) is substantially larger. The particle thus executes a lesser number of $Z$ oscillations (only five equatorial crossings as opposed to six in the initial case coded in blue). As mentioned above, the adiabaticity parameter $\kappa$ cannot be used in a straightforward manner here since the magnetic field line geometry is constantly changing. Still, were an instantaneous value of $\kappa$ to be considered, the $\mathrm{H}^{+}$behavior shown in red in Fig. 2 would correspond to a larger $\kappa$ value (larger field line curvature radius) and thus to a lower resonance order $N$ in Eq. (2) and, hence, a smaller number of $Z$ oscillations. In the lower right panel of Fig. 2, it can be seen that the test $\mathrm{H}^{+}$coded in red ultimately exits the neutral sheet with a substantial energy gain (up to $\sim 3 \mathrm{keV}$ ) and with a magnetic moment nearly identical to the initial one. For both trajectories (green and red), quasiadiabaticity thus persists despite the rapidly changing $B_{X}$ component in Eq. (3). In other words, Fig. 2 suggests that, depending upon phasing between midplane crossing and the surging electric field, particles may experience more and less significant energization and that this does not necessarily alter their Speiser behavior.

Figure 3 shows the results obtained considering a wider range of injection times. That is, test protons were launched from $Z=L$ with the same parameters as in Fig. 2 but by steps of $1 \mathrm{~s}$. Figure 3 shows the successive $Y$ values at $Z=0$ crossing as a function of equatorial crossing time, together with color-coded instantaneous energy. Here again, a $60 \mathrm{~s}$ relaxation (shaded area in Fig. 3) of the magnetic field lines was considered, yielding a peak dawn-to-dusk electric field of $\sim 0.3 \mathrm{mV} \mathrm{m}^{-1}$ at half collapse as illustrated in the upper panel of Fig. 3. It is apparent from the lower panel of Fig. 3 that test $\mathrm{H}^{+}$in the initial configuration experience six crossings of the $Z=0$ plane. As for test $\mathrm{H}^{+}$intercepting the midplane during the magnetic transition, two domains can be distinguished. The first domain consists of particles that cross the equator in the early stage of the reconfiguration. For these particles, $B_{\mathrm{t}}$ in Eq. (4) does not significantly differ from $B_{\text {pre-. }}$ These particles experience the largest energization (from $1 \mathrm{keV}$ up to $\sim 5 \mathrm{keV}$ ), and, as a result of their fast $Z$ oscillation (with timescale varying as $R_{\mathrm{C}} / V$ ), they may experience an additional crossing of the midplane while being turned back from $+X$ to $-X$ directions of propagation. The second domain in Fig. 3 consists of particles that cross the equator in the late stage of the reconfiguration. Although these latter particles do experience some energization as well, magnetic field lines have now turned significantly more dipolar so that the timescale of the $Z$ oscillation is larger (due to a larger field line curvature radius) and the particles execute a smaller number of $Z$ oscillations. Ultimately, in the postdipolarization configuration, the test $\mathrm{H}^{+}$in Fig. 3 only experiences four crossings of the midplane, the smaller curvature of the magnetic field lines leading to a larger $\kappa$ and a smaller resonance order (viz., $N \approx 3$ in Eq. 2).

During the magnetic transition, increasing energy due to the induced electric field leads to faster $Z$ oscillation (because of larger $V$ ), while an increasing field line curvature radius due to dipolarization leads to slower $Z$ oscillation (because of larger $R_{\mathrm{C}}$ ). The combination of these two opposite effects can be better seen in Fig. 4, which presents selected parameters of the model trajectories in Fig. 3 as a function of the last equatorial crossing time. In the center panel of Fig. 4, it can be seen that the number of midplane crossings first temporarily increases from six to seven (note the corresponding gap obtained between 10 and $20 \mathrm{~s}$ because of the time required to execute an additional $Z$ oscillation) and then gradually decreases from seven to four. In the bottom panel of Fig. 4, it can be seen that the particle velocity (shown in red and normalized to its initial value) first increases faster than the field line curvature radius, which is responsible for a faster $Z$ oscillation and additional midplane crossing. Because energization subsequently decreases and the field line curvature radius (shown in blue and normalized to its initial value) increases, slower $Z$ oscillations are then obtained and, hence, a decreasing number of midplane crossings. Were an instantaneous $\kappa$ to be considered here (shown in black in Fig. 4 and normalized to its initial value), this would translate as a two-step response, with a $\kappa$ decrease followed by a $\kappa$ increase.

Figure 5 shows model trajectories that illustrate the successive increase and decrease in $Z$ oscillations depending upon phasing with the surging electric field. In the left panels of this figure, the equatorial crossing sequence is shown in the $Y-Z$ plane for different injection times, while the associ- 

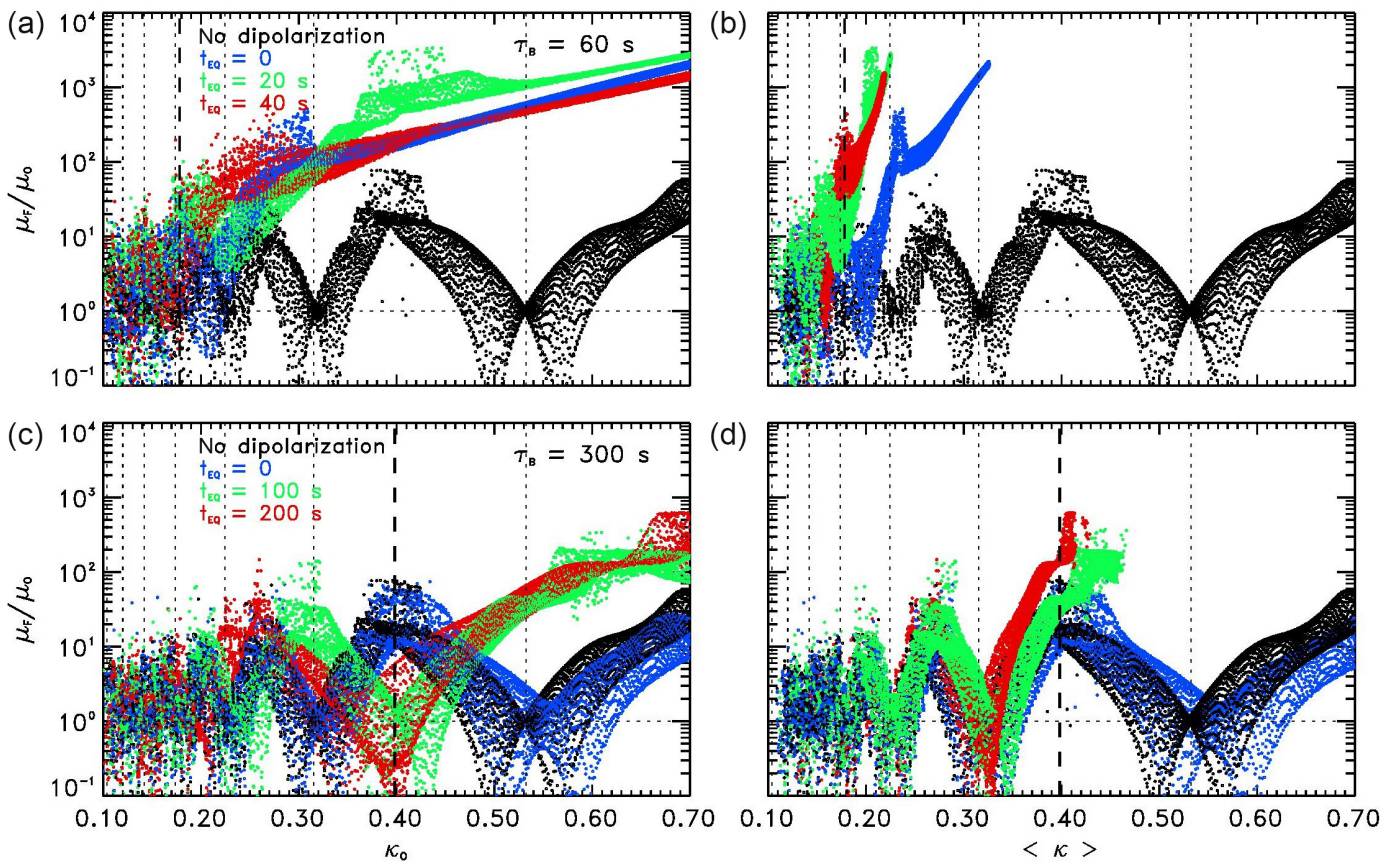

Figure 7. Final $\mathrm{H}^{+}$magnetic moment as a function of parameter $\kappa$ for different (color-coded) equatorial crossing times. A 60 (top) or $300 \mathrm{~s}$ (bottom) magnetic transition is considered. In the left and right panels, the initial $\kappa$ value and the average $\kappa$ value at equatorial crossing are considered, respectively. The dashed vertical line shows the $\kappa$ value corresponding to the peak $\boldsymbol{E} x \boldsymbol{B}$ drift speed (denoted by $\kappa_{\boldsymbol{E}} x \boldsymbol{B}$ in the text). Vertical dotted lines show the expected energy resonances (Eq. 2).

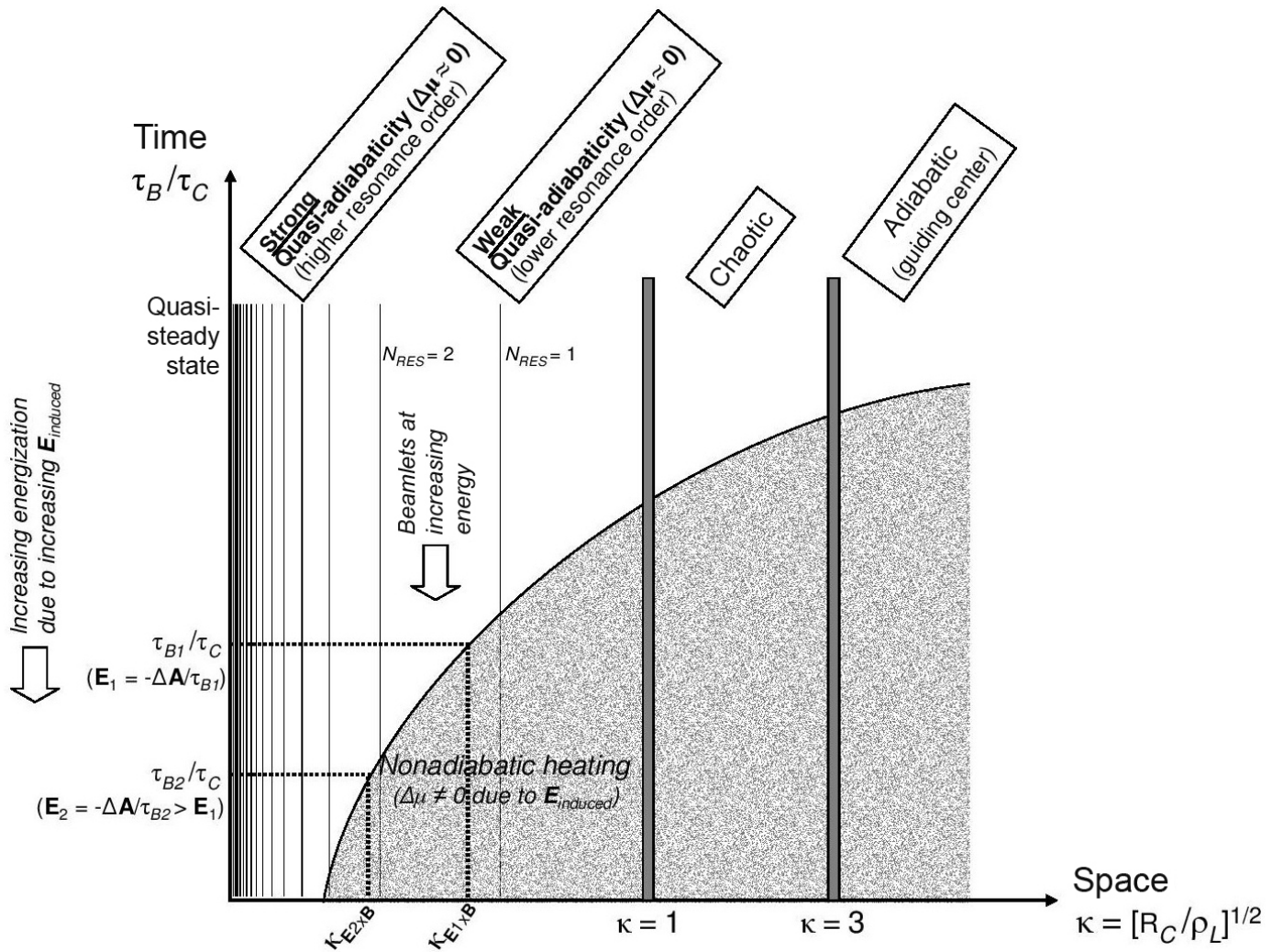

Figure 8. Schematic representation of quasi-adiabaticity evolution depending upon timescale of the magnetic field line reconfiguration. 
(a)

Z

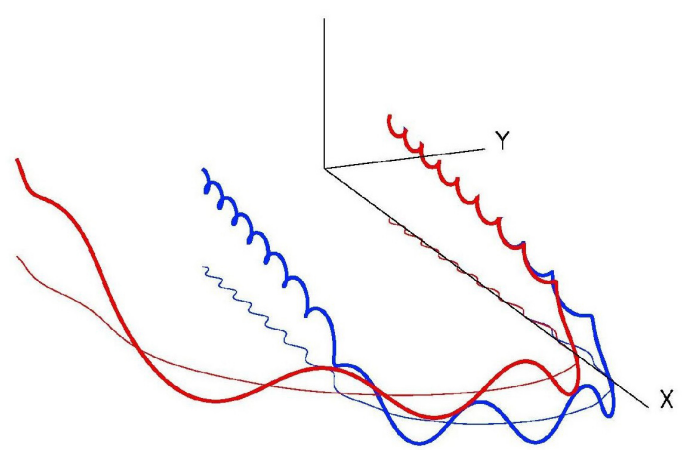

(c)

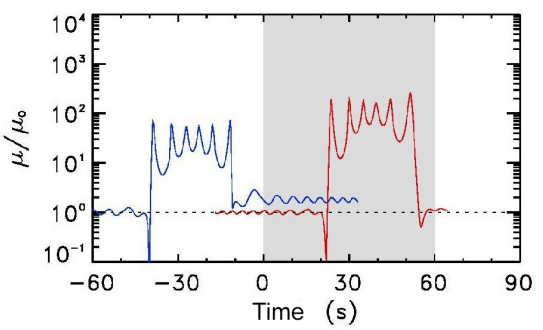

(b)

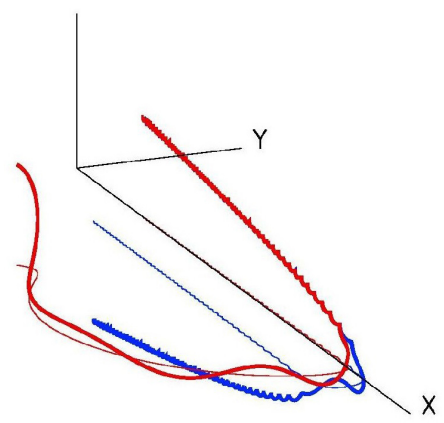

(d)

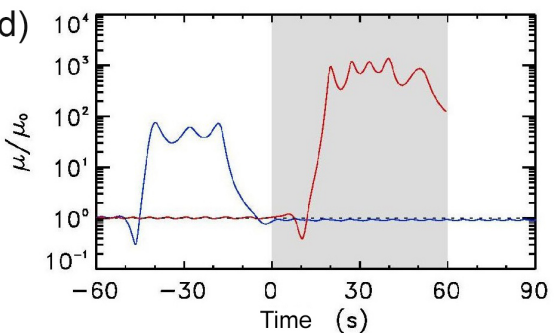

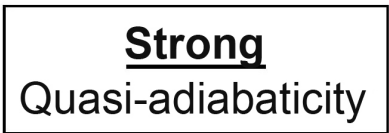

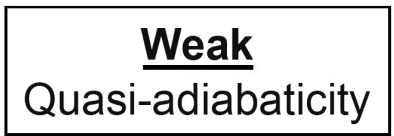

Figure 9. Model $\mathrm{H}^{+}$orbits illustrating (left) the case of "strong" quasi-adiabaticity and (right) the case of "weak" quasi-adiabaticity. Top and bottom panels show three-dimensional views of the $\mathrm{H}^{+}$trajectories and magnetic moment versus time, respectively. The test protons coded in blue and in red cross the field reversal before and during dipolarization, respectively. Thin lines in the top panels show the trajectory projections in the equatorial plane. A $60 \mathrm{~s}$ magnetic transition is considered.

ated variations of the $\kappa$ parameter (normalized to its value in the pre-dipolarization configuration) are shown in the right panels. The top panel of Fig. 5 shows an example of particles in the first domain described above. That is, as the equatorial crossing time increases, the induced electric field becomes more intense; hence, there is larger particle energy, faster $Z$ oscillations (from six to seven midplane crossings), and larger $Y$ displacement as compared to the reference trajectory (shown in dark blue). In contrast, in the two lower panels of Fig. 5, particles intercept the midplane later during dipolarization. Although some energization is noticeable, it is apparent that these particles execute a smaller number of $Z$ oscillations, with an amplitude that is either larger or smaller than that of the reference trajectory (dark blue). In the right panels of Fig. 5, distinct $\kappa$ variations can be seen depending upon equatorial crossing time, with a $\kappa$ decrease followed by a $\kappa$ increase.

\section{Discussion}

The aim of the present study is to examine how Speiser particles are affected by the surging electric field due to rapid reconfiguration of the magnetotail field lines. In other words, do quasi-adiabatic particles resist short-lived relaxation of a sharp field reversal? It is apparent from the above results that, although they are subjected to impulsive energization and exhibit different $Z$ oscillation sequences about the midplane, particles may indeed conserve their quasi-adiabatic character during such magnetic transitions. A more quantitative view of this result can be obtained from Fig. 6, which presents results of systematic computations for distinct (color-coded) resonance numbers and different initial times and gyration phases. Looking first at the top panel of this figure, which shows the $\mathrm{H}^{+}$energy at the exit of the field reversal as a function of equatorial crossing time, it can be seen that the net energy gain realized by the particles depends upon phasing with the induced electric field. Regardless of initial energy, maximum energization is obtained for particles that intercept the equator about $20 \mathrm{~s}$ after the onset of dipolarization. Also, 

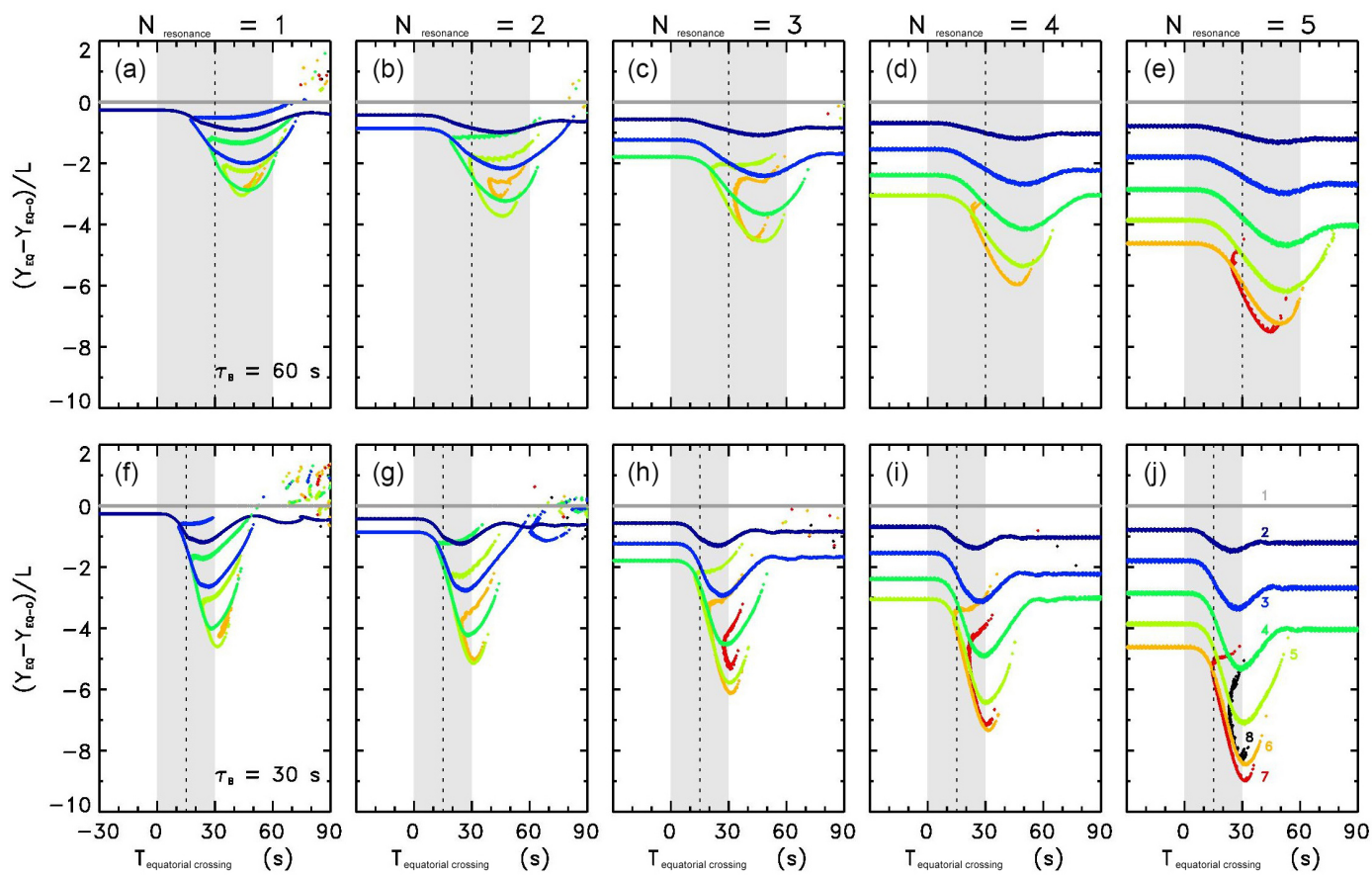

Figure 10. Identical to Fig. 3 but for different $\mathrm{H}^{+}$energy (from $N=1$ to $N=5$ from left to right panels) and for two different reconfiguration timescales (60 and $30 \mathrm{~s}$ in top and bottom panels, respectively). The equatorial crossing number is color-coded as indicated in the lower right panel.
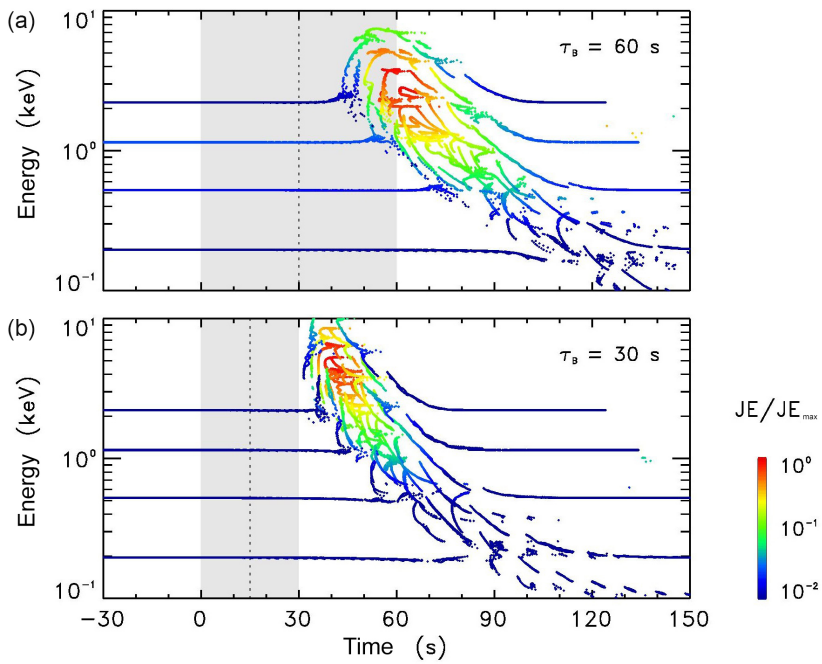

Figure 11. Computed $\mathrm{H}^{+}$energy-time spectrograms for two different reconfiguration timescales (60 and $30 \mathrm{~s}$ in top and bottom panels, respectively). The normalized energy flux is coded according to the color scale on the right.

it can be seen in this panel that particles launched with the lowest energy ( $N=1$ in Eq. 2 or, equivalently, an initial energy of $\sim 8 \mathrm{eV}$; coded in blue) may be systematically energized by up to $\sim 1 \mathrm{keV}$ as a result of nonadiabatic heating. A weaker energization is achieved for particles that inter- cept the field reversal in the late phase of dipolarization. The lower panel of Fig. 6 shows the particle magnetic moment at the exit of the field reversal, and it can be seen that particles with the lowest initial energy may experience $\mu$ enhancements of up to $\sim 3$ orders of magnitude. In contrast, particles with large initial energy (shown in red) experience much weaker $\mu$ variations. Note finally the magnetic moment modulation obtained depending upon equatorial crossing time or, equivalently, phasing with the surging electric field.

A more global view of the response of quasi-adiabatic particles to the changing field line geometry can be obtained from Fig. 7, which shows the final magnetic moment (normalized to the initial value) of test $\mathrm{H}^{+}$over a wide range of $\kappa$ values and for different (color-coded) equatorial crossing times. For comparison, top and bottom panels in this figure show the results obtained for distinct relaxation timescales (60 and $300 \mathrm{~s}$, respectively). The profiles in black in the different panels of this figure relate to the pre-dipolarization configuration. These profiles clearly display the resonance effect discussed above (Eq. 2) with a succession of large (typically, above 10) or small $\mu$ changes depending upon $\kappa$. Also, the vertical dashed-dotted line in each panel shows the $\kappa_{\boldsymbol{E} x \boldsymbol{B}}$ value corresponding to the peak $\boldsymbol{E} x \boldsymbol{B}$ drift speed during the magnetic transition. This $\kappa_{\boldsymbol{E} x \boldsymbol{B}}$ value is smaller in the upper panels as compared to the lower panels since a smaller reconfiguration timescale is considered (hence, there is a more pronounced peak electric field owing to Maxwell's equation, $\boldsymbol{E}=-\partial \boldsymbol{A} / \partial t$, and a larger $\boldsymbol{E} x \boldsymbol{B}$ drift speed). It is appar- 
ent here that, for initial $\kappa$ values greater than $\kappa_{\boldsymbol{E} x \boldsymbol{B}}$ (equivalently, initial $\mathrm{H}^{+}$speed smaller than the peak $\boldsymbol{E} x \boldsymbol{B}$ drift speed), significant $\mu$ enhancements are obtained during the magnetic transition and that the resonance modulation nearly vanishes. In contrast, for initial $\kappa$ smaller than $\kappa_{\boldsymbol{E} x \boldsymbol{B}}$, the post-dipolarization magnetic moments are in a range comparable to the initial ones so that the quasi-adiabatic character of the particles is conserved, at least partially.

It should be kept in mind here that because of the changing field line geometry (equivalently, changing field line curvature), the $\kappa$ parameter cannot be used in a straightforward manner. Still, it can be seen by a comparison of right and left panels in Fig. 7 that, if a parameter $\kappa$ averaged over successive equatorial crossings is used instead of the initial $\kappa$, the above numerical results are better organized. In particular, the threshold value $\langle\kappa\rangle \approx \kappa_{\boldsymbol{E} x \boldsymbol{B}}$ approximately delineates the domain where large $\mu$ enhancements occur as a result of dipolarization. It is also apparent that, for $<\kappa>$ smaller than $\kappa_{\boldsymbol{E} x \boldsymbol{B}}$, quasi-adiabaticity and resonances persist. In this latter regime, Speiser particles that originate from regions of strong $\boldsymbol{B}$ may return to such regions of strong $\boldsymbol{B}$ because of negligible $\mu$ change, which may lead to specific signatures at low altitudes as will be seen hereinafter.

The results displayed in Fig. 7 are schematically summarized in Fig. 8, which shows a domain of nonadiabatic heating (i.e., prominent $\mu$ enhancement, coded in grey) that extends toward smaller $\kappa$ values when the inductive electric field increases (equivalently, at smaller $\kappa_{\boldsymbol{E} x \boldsymbol{B}}$ ). The sketch in Fig. 8 builds on Fig. 7 of Delcourt and Martin (1999), which was obtained in steady state. Such a steady-state situation corresponds to the upper part of Fig. 8, with a field variation timescale $\tau_{B}$ much larger than the particle gyro-period $\tau_{C}$. The lower part of Fig. 8 shows the evolution obtained when fast variations of the magnetic field are considered. In particular, it is apparent from Fig. 7 that low resonance orders (e.g., $N=1$ or 2 in Eq. 2) may be significantly altered in the presence of an induced electric field while higher resonance orders remain unchanged. To take into account this effect of temporal nonadiabaticity, we are led to qualify the quasi-adiabatic behavior, which is originally a steady-state concept (Büchner and Zelenyi, 1989). In Fig. 8, we denote by "weak" quasi-adiabaticity the low resonance orders that are easily broken by an induced electric field and by "strong" quasi-adiabaticity the high resonance orders that persist regardless of this induced electric field. These two types of quasi-adiabatic behaviors can be better seen in Fig. 9, which shows model $\mathrm{H}^{+}$trajectories and associated magnetic moment variations in each case. The blue profiles in this figure present the pre-dipolarization behaviors, and the red profiles present the behaviors achieved during dipolarization. The right panels of Fig. 9 show the results obtained for the $N=2$ resonance. It is apparent here that, while $\mu$ change is negligible in the steady pre-dipolarization configuration, significant $\mu$ variation is obtained at the exit of the field reversal in the case of dipolarization, hence the denomination as weak quasi-adiabaticity. In contrast, in the left panels of Fig. 9, which show the results obtained for the $N=5$ resonance, it can be seen that both in the steady pre-dipolarization configuration and in the case of dipolarization, the magnetic moment at exit is nearly identical to that at entry, hence the denomination as strong quasi-adiabaticity.

It was shown in Fig. 5 that, because of the rapid energization of the particles under the effect of the induced electric field, the oscillation frequency in the $Z$ direction may significantly increase; hence, there is a wider drift of the particles in the $Y$ direction as compared to the steady-state situation. This can be further seen in Fig. 10, which shows the $Y$ locations of successive equatorial crossings as a function of equatorial crossing time for different initial energies (from $N=1$ in the leftmost panel to $N=5$ in the rightmost panel) and for two different dipolarization timescales (60 and $30 \mathrm{~s}$ in top and bottom panels, respectively). Looking first at the upper right panel, it can be seen that, in the pre-dipolarization configuration, test protons with $N=5$ experience six crossings of the midplane (as coded in blue in Fig. 2). For test $\mathrm{H}^{+}$ traveling through the field reversal during dipolarization, an additional midplane crossing may occur (as coded in green in Fig. 2). Finally, for test $\mathrm{H}^{+}$interacting with the field reversal after dipolarization, only four crossings are obtained because of the larger field line curvature radius (equivalently, larger $\kappa$ parameter and smaller $Z$ oscillation frequency). On the other hand, in the lower right panel of Fig. 10, the smaller reconfiguration timescale considered leads to an induced electric field with a larger peak magnitude and, hence, a larger energization realized and a $Z$ oscillation frequency that increases to such an extent that the test $\mathrm{H}^{+}$now experiences up to eight crossings of the midplane. As a matter of fact, it is apparent by a comparison of top and bottom panels in Fig. 10 that the net number of midplane crossings (equivalently, the net distance traveled in the $Y$ direction) increases with decreasing dipolarization timescale due to larger particle energization under the effect of the induced electric field.

Because particles may remain quasi-adiabatic during the magnetic transition (equivalently, their magnetic moment at the exit of the field reversal is nearly identical to that at entry), those originating from regions of a strong $\boldsymbol{B}$ field may return to such regions like in steady-state situations. Because of the impulsive energization due to the short-lived induced electric field, this may lead to specific signatures at low altitudes. This can be better seen in Fig. 11, which shows energy-time spectrograms expected from quasi-adiabatic particles at the exit of the field reversal, assuming two different reconfiguration timescales (60 and $30 \mathrm{~s}$ in top and bottom panels, respectively). Test protons were launched with energies corresponding to the $\kappa$ parameter of the first five resonances (Eq. 2), and the computed spectrograms were obtained by recording these particles at $|Z|=L$, the time to reach this $Z$ height being shown on the abscissa (with $t=0$ corresponding to the onset of dipolarization). It is apparent from Fig. 11 that, as a result of the magnetic transition, pro- 
nounced dispersion structures may be obtained. Not unexpectedly, the maximum flux in these dispersion structures occurs at an energy that increases with the peak induced electric field; i.e., for a given magnetic transition, it occurs at a larger energy for a smaller dipolarization timescale (note also the decreasing width of this dispersion structure for smaller timescales).

Several studies (e.g., Mauk, 1986; Hirahara et al., 1996) have examined the formation of dispersed ion structures as a result of dipolarization of the magnetic field lines. The structures examined in these studies were observed in the near-Earth plasma sheet or in the mid-tail, i.e., in regions where the $\mathrm{H}^{+}$motion may be regarded as adiabatic (i.e., $\kappa>3$ ). Dispersion structures in these studies likely follow from violation of the second adiabatic invariant (associated with bouncing motion along the field line) during dipolarization, this latter invariant being otherwise conserved in steady state (e.g., Konstantinidis and Sarris, 2015). In contrast, the structure portrayed in Fig. 11 results from particle dynamics in the distant tail where the guiding center approximation is not valid and the motion is quasi-adiabatic (i.e., $\kappa<1$ ). In other words, the dispersion signature in Fig. 11 is to be expected in the vicinity of the open-closed field line boundary, and it is actually reminiscent of the time-of-flight dispersed ion structures (TDIS) observed in the plasma sheet boundary layer, as illustrated for instance in Plate 4 of Sauvaud et al. (1999) or in Plate 1 of Sergeev et al. (2000), which show INTERBALL-Auroral data at low altitudes during substorms. The present results suggest that such characteristic signatures in the outer boundary layer are produced by ions with strong quasi-adiabaticity during localized relaxations of the magnetotail field reversal.

\section{Conclusions}

The simulations presented in this study were aimed at investigating the response of quasi-adiabatic (Speiser) particles to rapid reconfigurations of the magnetotail field lines. Such an examination is necessary since quasi-adiabaticity is a steadystate concept that prevents the use of a guiding-center-based description, and the timescale of the magnetic field line reconfiguration (of the order of a few minutes) may be comparable to that of the meandering sequences. These simulations demonstrate that, although the surging electric field induced by the dipolarizing field lines may lead to nonadiabatic heating, the quasi-adiabatic character of the particles may, at least partially, be conserved. Because of the rapidly changing field line geometry, the usual adiabaticity parameter $\kappa$ cannot be used to characterize the particle dynamics, but variations of the particle magnetic moment may be organized using a $\kappa$ parameter averaged over successive equatorial crossings. When this average $\kappa$ parameter exceeds the $\kappa$ parameter corresponding to the peak $\boldsymbol{E} x \boldsymbol{B}$ drift speed, prominent magnetic moment enhancements are obtained, an effect that we qualify as weak quasi-adiabaticity as opposed to strong quasi-adiabaticity (smaller $\kappa$ values), which persists despite the magnetic transition. Also, it appears that the particle dynamical behaviors may be organized into two categories, viz., (1) particles intercepting the sharp field reversal in the early stage of the relaxation that are subjected to an energization faster than the field line curvature change (hence, there are more numerous $Z$ oscillations and a wider drift in the $Y$ direction); (2) particles intercepting the field reversal in the late stage of the relaxation that are subjected to substantial energization as well but in a more dipolar configuration so that the $Z$ oscillation frequency decreases and the particle motion resembles that obtained at larger $\kappa$ values. Because of the rapid energization imparted by the surging electric field, strongly quasi-adiabatic particles during substorms may lead to characteristic energy-time dispersion structures upon traveling down to low altitudes, as is observed in the plasma sheet boundary layer.

\section{Data availability}

The data that are used in this paper and that are shown in the different figures are available from the first author upon request. Data that are referred to in this paper are available in cited references.

Acknowledgements. The work of Helmi V. Malova and Lev M. Zelenyi was carried out with financial support from grants 16-5216009 NCNIL_a and 16-02-00479 of the Russian Science Foundation.

The topical editor, E. Roussos, thanks two anonymous referees for help in evaluating this paper.

\section{References}

Aggson, T. L., Heppner, J. P., and Maynard, N. C.: Observations of large magnetospheric electric fields during the onset phase of a substorm, J. Geophys. Res., 88, 3981-3990, 1983.

Anderson, B. J., Decker, R. B., Paschalidis, N. P., and Sarris, T.: Onset of nonadiabatic particle motion in the near-Earth magnetotail, J. Geophys. Res., 102, 17553-17570, 1997.

Artemyev, A. V. and Vasiliev, A. A.: Resonant ion acceleration by plasma jets: Effects of jet breaking and the magnetic-field curvature, Phys. Rev. E, 91, 053104, doi:10.1103/PhysRevE.91.053104, 2015.

Artemyev, A. V., Liu, J., Angelopoulos, V., and Runov, A.: Acceleration of ions by electric field pulses in the inner magnetosphere, J. Geophys. Res., 120, 4628, doi:10.1002/2015JA021160, 2015.

Ashour-Abdalla, M., Zelenyi, L. M., Peroomian, V., Richard, R. L., and Bosqued, J. M.: The mosaic structure of plasma bulk flows in the Earth's magnetotail, J. Geophys. Res., 100, 19191-19210, 1995.

Birn, J., Artemyev, A. V., Baker, D. N., Echim, M., Hoshino, M., and Zelenyi, L. M.: Particle acceleration in the magnetotail and aurora, Space. Sci. Rev., 173, 49-102, 2012. 
Büchner, J. and Zelenyi, L. M.: Regular and chaotic charged particle motion in magnetotaillike field reversals: 1, Basic theory of trapped motion, J . Geophys. Res., 94, 11821-11842, 1989.

Burkhart, G. R. and Chen, J.: Differential memory in the Earth's magnetotail, J. Geophys. Res., 96, 14033-14049, 1991.

Cary, J. R. and Brizard, A. J.: Hamiltonian theory of guiding center motion, Rev. Mod. Phys., 81, 693-738, 2009.

Chen, J. and Palmadesso, P. J.: Chaos and nonlinear dynamics of single particle orbits in magnetotaillike magnetic field, J. Geophys. Res., 91, 1499-1508, 1986.

Delcourt, D. C. and Martin Jr., R. F.: Pitch angle scattering near energy resonances in the geomagnetic tail, J. Geophys. Res., 104, 383-394, 1999.

Delcourt, D. C., Sauvaud, J.-A., and Pedersen, A.: Dynamics of single-particle orbits during substorm expansion phase, J. Geophys. Res., 95, 20853-20865, 1990.

Delcourt, D. C., Sauvaud, J.-A., Martin Jr., R. F., and Moore, T. E.: On the nonadiabatic precipitation of ions from the near-Earth plasma sheet, J. Geophys. Res., 101, 17409-17418, 1996.

Hirahara, M., Mukai, T., Nagai, T., Kaya, N., Hayakawa, H., and Fukunishi, H.: Two types of ion energy dispersions observed in the nightside auroral regions during geomagnetically disturbed periods, J. Geophys. Res., 101, 7749-7767, 1996.

Konstantinidis, K. and Sarris, T.: Calculations of the integral invariant coordinates $I$ and $L^{*}$ in the magnetosphere and mapping of the regions where $I$ is conserved, using a particle tracer (ptr3D v2.0), LANL*, SPENVIS, and IRBEM, Geosci. Model Dev., 8, 2967-2975, doi:10.5194/gmd-8-2967-2015, 2015.

Malova, H. V., Popov, V. Y., Delcourt, D. C., Petrukovich, A. A., and Zelenyi, L. M.: Antisunward structure of thin current sheets in the Earth's magnetotail: Implications of quasi-adiabatic theory, J. Geophys. Res., 118, 4308-4318, 2013.

Miyashita,Y., Machida, S., Kamide, Y., Nagata, D., Liou, K., Fujimoto, M., Ieda, A., Saito, M. H., Russell, C. T., Christon, S. P., Nosé, M., Frey, H. U., Shinohara, I., Mukai, T., Saito, Y., and Hayakawa, H.: A state-of-the-art picture of substormassociated evolution of the near-Earth magnetotail obtained from superposed epoch analysis, J. Geophys. Res., 114, A01211, doi:10.1029/2008JA013225, 2009.

Nosé, M., Takahashi, K., Keika, K., Kistler, L. M., Koga, K., Koshiishi, H., Matsumoto, H., Shoji, M., Miyashita, Y., and Nomura, R.: Magnetic fluctuations embedded in dipolarization inside geosynchronous orbit and their associated selective acceleration of $\mathrm{O}^{+}$ions, J. Geophys. Res., 119, 4639, doi:10.1002/2014JA019806, 2014.
Northrop, T. G.: The Adiabatic Motion of Charged Particles, Wiley Intersci., New York, 1963.

Reeves, G. D., Henderson, M. G., McLachlan, P. S., and Belian, R. D.: Radial propagation of substorm injections, ESA SP-389, 579 pp., 1996.

Runov, A., Angelopoulos, V., Sitnov, M. I., Sergeev, V. A., Bonnell, J., McFadden, J. M., Larson, D., Glassmeier, K.H., and Auster, U.: THEMIS observations of an earthwardpropagating dipolarization front, Geophys . Res. Lett., 36, L14106, doi:10.1029/2009GL038980, 2009.

Sarris, T. E., Li, X., Tsaggas, N., and Paschalidis, N.: Modeling energetic particle injections in dynamic pulse fields with varying propagation speeds, J. Geophys. Res., 107, 1033, doi:10.1029/2001JA900166, 2002.

Sauvaud, J.-A., Popescu, D., Delcourt, D. C., Parks, G. K., Britmacher, M., Sergeev, V., Kovrazhkin, R. A., Mukai, T., and Kokubun, S.: Sporadic plasma sheet ion injections into the highlatitude auroral bulge: Satellite observations, J. Geophys. Res., 104, 28565-28586, 1999.

Sergeev, V. A., Sazhina, E. M., Tsyganenko, N. A., Lundblad, J. A., and Soraas, F.: Pitch-angle scattering of energetic protons in the magnetotail current sheet as the dominant source of their isotropic precipitation into the nightside ionosphere, Planet. Space Sci., 31, 1147-1155, 1983.

Sergeev, V. A., Sauvaud, J.-A., Popescu, D., Kovrazhkin, R. A., Lutsenko, V. N., Zelenyi, L. M., Syrjasuo, M., Viljanen, A., Pulkkinen, T. I., Kudelas, K., Kokubun, S., and Mukai, T.: Plasma sheet ion injections into the auroral bulge: Correlative study of spacecraft and ground observations, J. Geophys. Res., 105, 1846518482, 2000.

Speiser, T. W.: Particle trajectories in model current sheets, 1. Analytical solutions, J. Geophys. Res., 70, 4219-4226, 1965.

Sundberg, T., Slavin, J. A., Boardsen, S. A., Anderson, B. J., Korth, H., Ho, G. C., Schriver, D., Uritsky, V. M., Zurbuchen, T. H., Raines,J. M., Baker, D. N., Krimigis, S. M., McNutt Jr., R. L., and Solomon, S. C.: MESSENGER observations of dipolarization events in Mercury's magnetotail, J. Geophys. Res., 117, A00M03, doi:10.1029/2012JA017756, 2012.

Ukhorskiy, A. Y., Sitnov, M. I., Merkin, V. G., and Artemyev, A. V.: Rapid acceleration of protons upstream of earthward propagating dipolarization fronts, J. Geophys. Res., 118, 4952-4962, doi:10.1002/jgra.50452, 2013.

Zhou, X.-Z., Angelopoulos, V., Sergeev, V. A., and Runov, A.: Accelerated ions ahead of earthward propagating dipolarization fronts, J. Geophys. Res., 115, A00I03, doi:10.1029/2010JA015481, 2010. 